\title{
Proactive - Adaptive Ramp Metering Scheme for Urban Highways
}

\section{Mandar Khanal*}

Department of Civil Engineering, Boise State University, USA

Ramp metering is a proven, effective measure to deal with congestion and accidents. The most comprehensive study ever conducted on ramp metering was published in 2001 and evaluated their effectiveness by turning off the meters in the Minneapolis-St. Paul area [1]. Without meters, freeway travel times increased $22 \%$ resulting in 2.6 million hours of unexpected delay. Speeds were also found to increase and speed variability increased in the absence of metering, peak period crashes on previously metered freeways and ramps increased by $26 \%$, resulting in annual savings of 1,041 crashes or approximately four crashes per day. According to the ITS Deployment Tracking 2010 Survey Results, ramp metering and adaptive signal controls received high benefit rankings by agencies that have adopted them [2].

Travel time related measures, as perceived by drivers, is arguably the most indicative measure of a facility's quality of service. Network coordinated ramp metering has the potential to increase throughput, reduce travel time, reduce accidents, and reduce emissions. Simulation can be utilized to test performance measures for various ramp metering schemes and compare the results with the current no metering scenario. Ramp metering is a proactive solution that is cost-effective since it utilizes existing infrastructure with the bulk of the costs relating to traffic signals.

Ramp metering has been implemented throughout the United States; however, most schemes are not adaptive. The rate at which vehicles are allowed to enter the highway from on-ramps, which is known as the metering rate, is based in most cases on the operator's experience about congestion on the road sections under their jurisdiction. There are more sophisticated schemes, which are adaptive, but they are reactive in the sense that they are adapting to conditions that existed some time period ago. In a truly reactive-adaptive system the expected traffic conditions on the highway in a future time period needs to be continuously forecasted and the ramp metering rate adjusted to conditions that are expect to occur in that time period. Such a system will be proactive and if this is accomplished in real-time, the resulting system will be a proactive-adaptive system. Such a system does not currently exist anywhere in the world.

The improved ramp metering scheme described above can be accomplished through a two-step extended Kalman Filter forecasting of traffic state on the main highway combined with a control system based upon that state. The traffic state is the current speed, flow, and density conditions as measured by loop detectors or similar devices. Forecasting is accomplished with an Extended Kalman filter (EKF) that works by making a prediction of the future traffic state and comparing the estimate with real-time measurements. The EKF is a recursive data processing algorithm, meaning predictions are based on only the previous time-steps prediction so the filter does not require all previous data to be stored and repossessed. This allows for low computational cost and on-line forecasting.

Initial work in developing such a ramp metering scheme at the Eagle Road Interchange on I-84 in Boise, Idaho has been completed. Six radar sensors were deployed on the east bound and west bound directions and collected 5-minute average data on speed, volume, occupancy, and vehicle classification. A computer model of the I-84 corridor from Ten Mile interchange to the Cloverdale Road overpass using Vissim microsimulation software was developed. Algorithms for a control scheme that uses the forecast of the traffic state to compute the optimal metering rate for the two on-ramps in the east bound direction are being developed in MATLAB and will be tested in the Vissim model. Modelling and simulation will be expanded to test the effects of a coordinated network.

The long-term goal of this effort is to extend the implementation of the proactive-adaptive ramp metering scheme to other locations in the state that are experiencing growing congestion. System-wide improvements in travel time and reduction in delays will result with proactive-adaptive ramp meters at these locations. Such improvements can be disseminated through Traveler Information Systems to inform the public about estimated time of travel, specifically during incidents or when winter weather affects travel conditions. Implementation of dynamic or variable speed limits is also another potential application.

\section{References}

1. Twin Cities Ramp meter evaluation, Final Report (2001) Cambridge Systematics, Inc. Minnesota Department of Transportation.

2. Deployment of ITS: A Summary of the 2010 National Survey Results (2011) RITA Intelligent Transportation systems Joint Program Office.
*Corresponding author: Mandar Khanal, Department of Civil Engineering, Boise State University, USA, Tel: +1 208-426-1153; E-mail: mkhanal@boisestate.edu

Received April 01, 2016; Accepted April 02, 2016; Published April 04, 2016

Citation: Khanal M (2016) Proactive - Adaptive Ramp Metering Scheme for Urban Highways. J Civil Environ Eng 6: e120. doi:10.4172/2165-784X.1000e120

Copyright: (ㅇ 2016 Khanal M. This is an open-access article distributed under the terms of the Creative Commons Attribution License, which permits unrestricted use, distribution, and reproduction in any medium, provided the original author and source are credited. 